\title{
Study of Strain characterization and microstructure evolution under compression stress in $\mathrm{Al}-\mathrm{Li}$ alloy
}

\author{
Xiao Jin $^{1,2}$ a , GuoDong Zhang ${ }^{1}$, Fei Xue ${ }^{1}$ and YanFen Zhao ${ }^{1}$ \\ 1 Suzhou Nuclear Power Research Institute, Suzhou 215004, PR China; \\ 2 Laboratory of Advanced Materials, School of Material Science and Engineering, Tsinghua \\ University, Beijing 100084, PR China. \\ jin_xiao2002@163.com
}

Keywords: Al-Li alloy, strain, microstructure, DIC.

\begin{abstract}
The strain variation and microstructure evolution of 2060-T8 alloy during bending are investigated by Micro-hardness point method (MHPM) and Digital Image Correlation (DIC), electron backscattered diffraction (EBSD). Experimental result shows DIC method can be used to characterize the strain micro-region on average and it can also represent the local strain tensor in the micro-region compared to the traditional MHPM. The strain is unevenly distributed in grains due to the mis-orientation between the adjacent grains difference, the mis-orientation has a blocking effect on the slip of dislocations during deformation in polycrystalline sample. The result shows the mis-orientation between adjacent grains is smaller with larger strain, while the mis-orientation between adjacent grains is larger with smaller strain. The degree of grain division in the process of deformation is related to the strain in this region, a new microstructure such as band structure is observed in grains with larger strain.
\end{abstract}

\section{Introduction}

The 2060 alloy is one of the third-generation Al-Li products, which is widely used for aerospace structures due to its excellent properties of high strength and low density[1-3]. The key step of alloy working as aircraft structural component is obtaining appropriate size and shape for aircraft structural component, and one commonly used deformation processing is bending, which is an environment-friendly and low waste method in the manufacture of structural parts[4, 5].

It is regarded that the outer line of specimen is under tensile stress and inner line of specimen is under compressive stress during bending[6,7]. Lots of work have been carried in the microstructure evolution under uniaxial tensile stress, the results show typical band structure are observed which is defined as geometrically necessary boundaries (GNBs)[8-11]. This microstructure evolution has a very significant impact on mechanical properties[12-14]. The microstructures and strain evolution of the tensile stress region are studied so much is due to fact that the region is easy to crack under tensile stress, and the region under compressive stress is not easy to crack during the deformation, so it is relatively less for its research.

In the present study, we aimed to find a quasi-in situ experimental method combined with strain measurement and microstructure characterization during bending, and the microstructure evolution are studied further more. It is important to understand the microstructure evolution under compressive stress because it greatly influences the mechanical properties of 2060 alloy and results of its research hardly can been found at present.

\section{Experimental}

In the present work, the material used for this study was a commercially 2060 aluminum alloy made by Alcoa Inc., the heat treatment is T8. In this study, T8 heat treatment of interested material is 
identified as: solution heat treatment at a temperature about $500{ }^{\circ} \mathrm{C}$ in accordance with AMS2722, during which the grains near the surfaces are recrystallized and artificial aging has been performed at $(143 \pm 2.8){ }^{\circ} \mathrm{C}$ for $35 \mathrm{~h}$. The typical microstructure of 2060-T8 alloy is shown in Fig.1[15], from picture the pancake-shaped grain structure with partially recrystallization, in rolling direction the grains are significantly flattened and elongated, and the grain dimensions in surfaces are comparably larger than those in center of the sheet. During the experiment process, specimen is bent to $10 \mathrm{~mm}$, $5 \mathrm{~mm}$ and 3mm, respectively, and relevant test are carried out under the different bend radius condition.

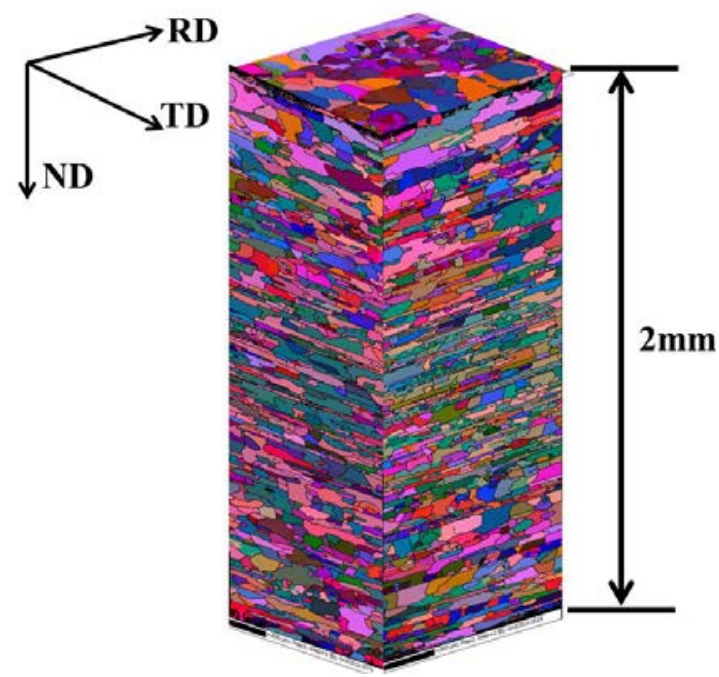

Fig. 1 Triplanar EBSD micrograph of 2060-T8 alloy

The composition of the of the studied alloy analyzed by infrared spectrometer, and the element content (wt.\%) is follow: $\mathrm{Ag}(0.34), \mathrm{Cu}(3.52), \mathrm{Fe}(0.03), \mathrm{Mg}(0.66), \mathrm{Mn}(0.29), \mathrm{Si}(0.02), \mathrm{Ti}(0.03)$, $\operatorname{Zn}(0.34), \operatorname{Zr}(0.1)$, Li(0.73), Al(Rest).

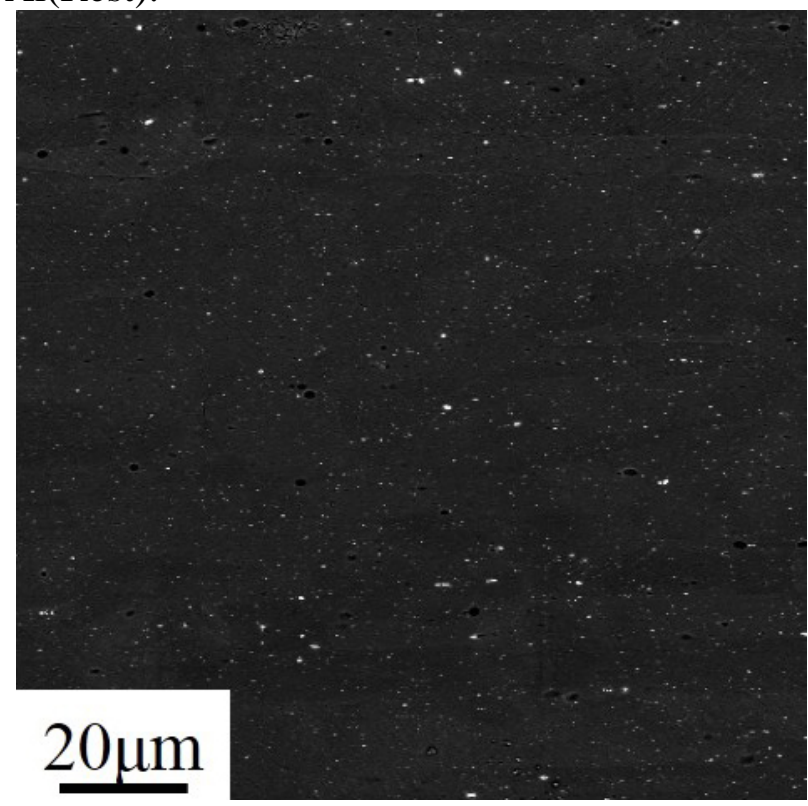

Fig.2 Microstructure of 2060 alloy with precipitates distribution in a BSE image

EBSD data collected by Oxford Nordly 2, Accelerating voltage of $20 \mathrm{kV}$, magnification of 300× and a working distance of $15 \mathrm{~mm}$ were used to scan over the same area of SEM scan, step size is $2 \mu \mathrm{m}$.

The DIC technology is used to characterize the strain evolution of interested region during deformation, and data are recorded during different stages of deformation[16-18]. The data of DIC require contain features obviously, as shown in Fig.2. In this map, a large number of precipitates (white points in Fig.2) with different sizes are defined as random speckle patterns to record the corresponding displacements. The precipitates randomly dispersed within the grains are evident, the precipitates appear as speckle patterns for strain calculations. Then these similar maps at different 
deformation stages collected by BSE probe are used as an input data to a DIC system for strain calculations.

\section{Results and discussion}

(1) Traditional method of strain measurement
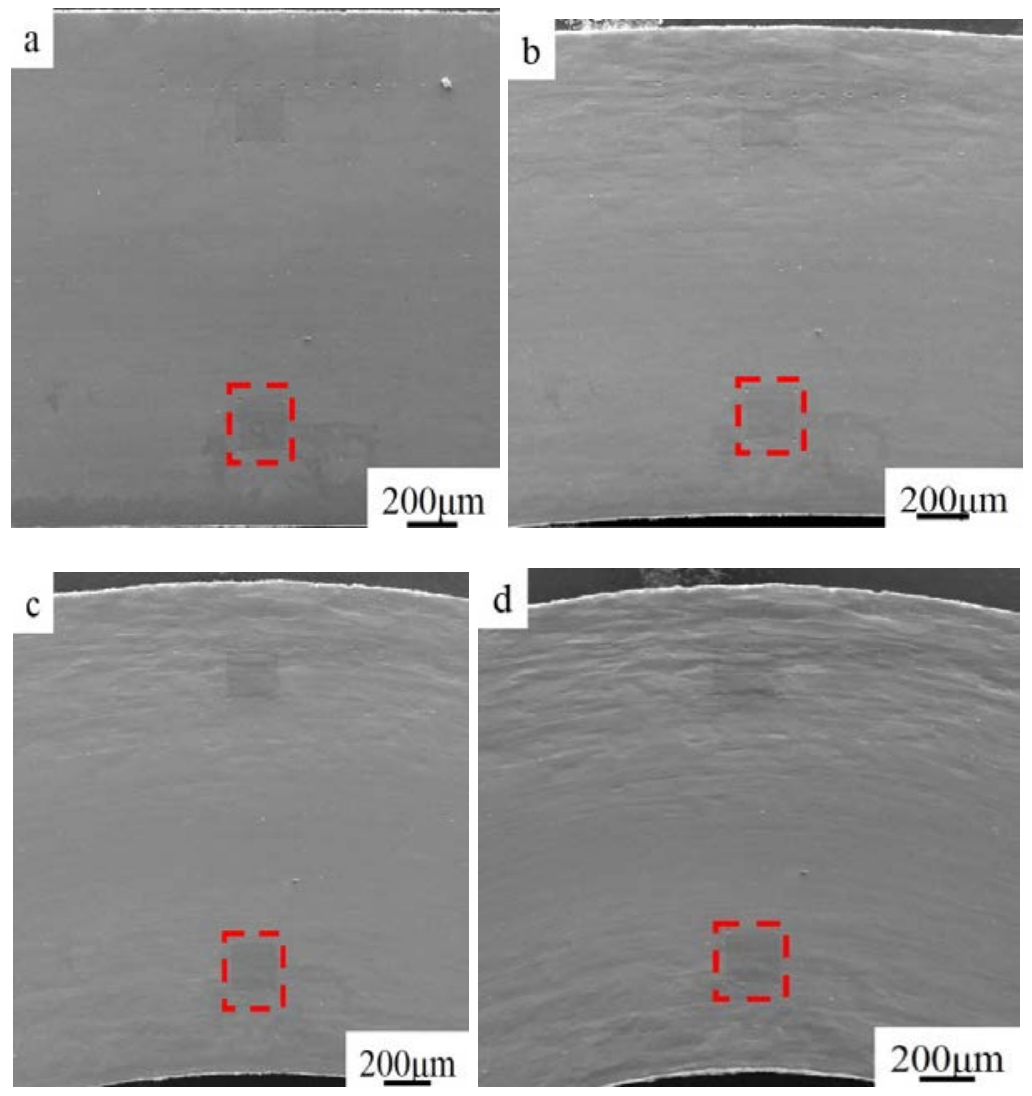

Fig.3 Morphology of simple with different bend radius: (a) unbend; (b) $r=10 \mathrm{~mm}$; (c) $r=5 \mathrm{~mm}$; (d) $\mathrm{r}=3 \mathrm{~mm}$

Before the DIC technology applied in characterization of surface strain, the Micro Hardness Point Method (MHPM) is one of the most commonly method in this area[19]. The principle of MHPM is according to displacement of gradient $F$ at coordinate points round the interested region during deformation and calculate the strain tensor during deformation.

In this experiment, region near the inner line of bent simple is followed, as the red dotted line shown in Fig.3. The surface of specimen embossed obviously during the bending in images. The micro hardness points are made at the four corners of red dotted rectangle as feature points, then specimen is bent to $10 \mathrm{~mm}, 5 \mathrm{~mm}$ and $3 \mathrm{~mm}$ respectively. The relative coordinates of four micro hardness points are record during deformation, then the average amount of strain in this region is calculated, as shown in Fig.4

Fig. 4 shows strain changed in the red dotted line region during bending, and three strain tensors $\left(\varepsilon_{\mathrm{xx}}, \varepsilon_{\mathrm{xy}}, \varepsilon_{\mathrm{yy}}\right)$ are studied. The major strain tensor $\varepsilon_{\mathrm{xx}}$ increased rapidly during bending, the value of Exx reach 0.14 when the bend radius near $3 \mathrm{~mm}$. The strain tensor of Eyy increase with the decrease of the bend radius, while the variation range is small. The strain tensor of $E x y$ almost unchanged during deformation. From the results of variation in strain tensors, it is concluded the strain in interest region can be equivalent treated as in the process of uniaxial compression. 


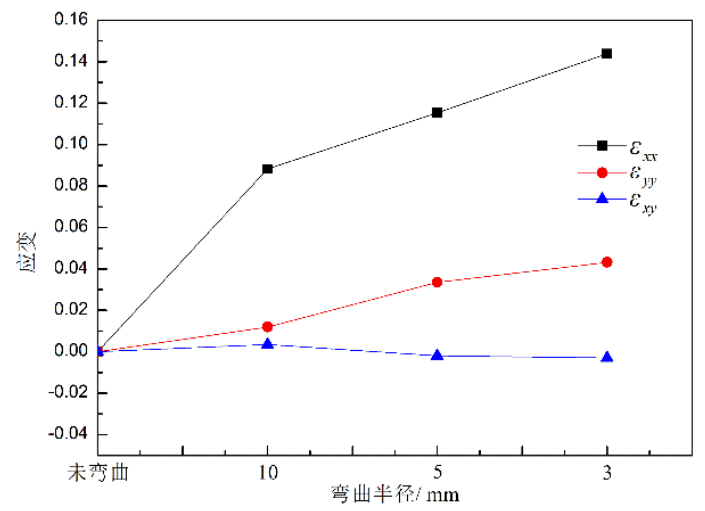

Fig.4 The strain changed during bending

The MHPM can characterize a region of the average strain changes effectively, but it is hard to show the strain of micro-region, because there are have not enough features for tracking and calculation, it is inadequate in analysis the micro-region microstructure evolution combined with EBSD result.

(2) Strain measurement and microstructure evolution

(2.1) DIC method of strain measurement
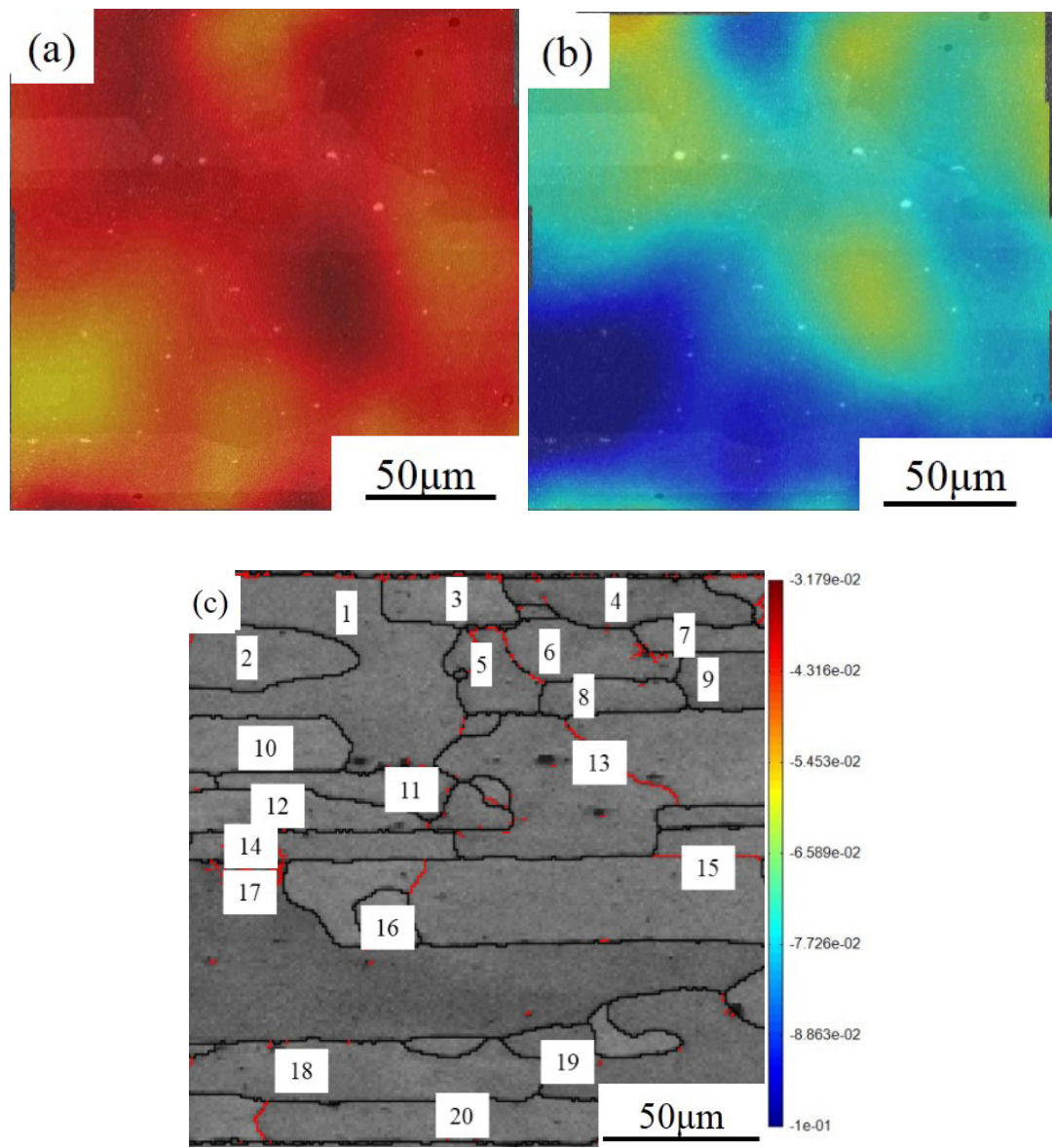

Fig.5 The cloud map of strain during bending under different radius:

(a) $r=10 \mathrm{~mm}$, (b) $r=5 \mathrm{~mm}$, (c) EBSD map of interested region before deformation.

The DIC technology can meet the requirements of strain evolution calculation in micro-region, and even a particular region of the strain changes can be reflected by this method in case there are enough feature points inside. In this work, a large number of random dispersal precipitation phases in the material meet this demand well, there are two completely different colors in the BSE images(the matrix is black and precipitates is white) . 
Fig. 5 shows the interested region with cloud map of strain distribution under different bend radius and EBSD map with grains distribution before deformation. Fig.5(a) and Fig.5(b) show the strain distribution when the bend radius reach $10 \mathrm{~mm}$ and $5 \mathrm{~mm}$ (strain distortion due to surface float seriously when the bend radius reach $3 \mathrm{~mm}$ ), a general version of result that the amount of strain increase with the bend radius decreased. Compared with Fig.5(c), it is found that train distribution is not consistent of all locations in the interest region, which can be determined that the MHPM is inaccurate reflect the strain in all locations in interested region (the darker blue mean larger strain and the darker red mean smaller strain). In addition, strain in every grain also is different from others. There are obvious strain concentration on some grain, and strain in different grains are also significantly different with the bend radius reduced. For example, when the bend radius is $5 \mathrm{~mm}$, the strain concentration mainly occurs in regions such as grain 1 and grain3, and the other micro-region of strain concentration is near grain 17.

It is believed that the main strain tensor is $\varepsilon_{x x}$ in interested region from Fig.4. That is, the interested region can be approximately seem as being deformed under uniaxial compressive stress during the bending process. Therefore, Schmid factor analysis is a common method in grain strain capacity under uniaxial compressive stress[9, 20, 21]. Fig. 6 shows the Schmid factor analysis of each grain in interested region under the uniaxial compressive stress. Grains with larger Schmitt factors are grain6, grain8 and grain13 as shown in Fig. 6, which mean these grains are easier to have deformation. In the case of uniaxial compressive stress, grains with larger strain is not the above-mentioned grains, but across multiple grain micro-regions. Combined with Fig.5, it can be seen that although the Schmid factor of grain3, grain5, grain15 and grain17 is not the largest, the amount of strain in these grains are larger relatively.

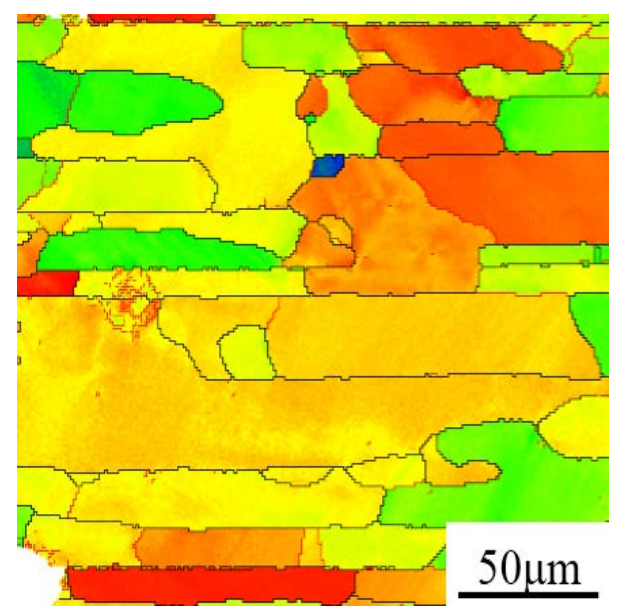

Fig.6 The distribution of Schmidt factors before deformation

Another consideration of uneven strain distribution is that the mis-orientation of grains in the polycrystals[22]. Table 1 shows the grains' Euler angle and mis-orientation between adjacent grains in interested region. Combined with Fig.5 and Table 1, it is found that strain concentration is closely related to the mis-orientation between adjacent grains. For example, it is found that the strain concentration occurred between grain3 and grain5, while the mis-orientation between two grains is $11.8^{\circ}$. Another micro-region of strain concentration is round grain17, it is found that mis-orientation between grain 17 and round grains are small, mis-orientation between grain 17 and grain 14 is $29^{\circ}$, mis-orientation between grain 17 and grain 18 is $26^{\circ}$, mis-orientation between grain 17 and grain 15 is $31.4^{\circ}$. The result show the smaller mis-orientation between grains are easier to have larger deformation. In addition, the size of the grain 17 is much larger than other grains, the strength of the 
grain is lower than grain boundary relatively at room temperature, so that the grain is easier to be deformed than grain boundary, maybe it is another reason for grain 17 have larger strain.

In the region with smaller strain, it is found that the mis-orientation between grains are larger. Most micro-region s round grain1 have smaller strain, because the mis-orientation between grain1 and round grains are larger, the mis-orientation between grain 1 and grain 2 is $47.2^{\circ}$, mis-orientation between grain 1 and grain 3 is $53.6^{\circ}$, mis-orientation between grain 1 and grain 10 is $51.3^{\circ}$, mis-orientation between grain 11 and grain 5 even reach $59.2^{\circ}$. Most of the regions with smaller strain distribution have smaller mis-orientation between grains, but there are exceptions, there is no significant strain concentration between grain 4 and grain7, while the mis-orientation between these two grains is $18.7^{\circ}$. The reason for this phenomenon may be that the strain in this region is limited due to round grains have smaller strain.

Table 1 All grains’ Euler angle and mis-orientation between adjacent grains in interested region.

\begin{tabular}{|c|c|c|c|}
\hline Arain & Euler angle $/^{\circ}$ & Adjacent grains & Mis-orientation $/^{\circ}$ \\
\hline grain1 & $35.31,36.42,24$ & grain1-grain2 & 47.2 \\
\hline grain2 & $282.32,45,22.05$ & Grain1-grain3 & 53.6 \\
\hline grain3 & $32.71,89.52,37.76$ & grain1-grain10 & 51.3 \\
\hline grain4 & $208.86, \quad 35.33,53.89$ & Grain1-grain11 & 56.1 \\
\hline grain5 & $299.6,27.06,88.13$ & grain1-grain5 & 59.2 \\
\hline grain6 & $287.88,30.16,86.63$ & grain1-grain13 & 54.2 \\
\hline grain7 & 221.85,43.66,29.27 & grain1-grain12 & 55.7 \\
\hline grain8 & $125.24,42.86,57.7$ & grain3-grain4 & 55.2 \\
\hline grain9 & $312.22,44.75,68.59$ & grain3-grain5 & 11.8 \\
\hline grain10 & $65.84,52.43,46.08$ & grain4-grain6 & 42.3 \\
\hline grain11 & 93.37,45.97,20.3 & grain4-grain7 & 18.7 \\
\hline grain12 & $153.34,45.21,14.22$ & grain5-grain6 & 13.4 \\
\hline grain13 & $174.08,25.66,15.29$ & grain5-grain13 & 48.2 \\
\hline grain14 & $211.23,44.68,40.33$ & grain6-grain7 & 45.7 \\
\hline grain15 & $317.13,44.59,56.31$ & grain6-grain8 & 31.4 \\
\hline grain16 & $39.75,50.44,66.49$ & grain7-grain9 & 35.3 \\
\hline grain17 & 67.2,31.04,60.99 & grain8-grain13 & 31.3 \\
\hline grain18 & $202.88,44.49,7.34$ & grain9-grain13 & 56.8 \\
\hline grain19 & $191.57,40.99,14.92$ & grain10-grain 11 & 43.7 \\
\hline \multirow[t]{11}{*}{ grain20 } & 203.33,45.05,38.79 & grain11-grain12 & 55.7 \\
\hline & & grain12-grain14 & 45 \\
\hline & & grain14-grain15 & 56.31 \\
\hline & & grain14-grain17 & 29 \\
\hline & & grain14-grain15 & 24.2 \\
\hline & & grain15-grain16 & 31.4 \\
\hline & & grain16-grain17 & 30.4 \\
\hline & & grain17-grain18 & 26 \\
\hline & & grain18-grain19 & 8.5 \\
\hline & & grain18-grain20 & 31.8 \\
\hline & & grain19-grain20 & 33.7 \\
\hline
\end{tabular}

The reason for the close relationship of mis-orientation and strain concentration is that the grain boundary has a blocking effect on the slip of the dislocations. The dislocation moves in the polycrystals in the process of deformation of the polycrystalline sample[21], the grain boundary has some resistance to the slip of the dislocations due to the grain orientation difference on both sides of the grain boundary, when the mis-orientation is large dislocation is difficult to slip from grain into the second grain. Multiple slip systems need to start at the same time in order to meet the grain boundary deformation of the coordination. This also leads to dislocations that are difficult to pass through the grain boundary and block at the grain boundary, which increasing the strength of micro-region and making micro-region hard to deformation [23, 24]. 


\section{(2.2) Microstructure evolution during bending}

Fig.7 show the microstructure evolution at interested micro-region under different bend radius. From Fig.7(a)-(c), the small angle grain boundary(SAGB) in grains increased rapidly with bend radius decreased, it can be indicated that the grain in interested micro-region have a significant plastic deformation during bending. In Fig.7(c), the band structure can be observed obviously, the traces are analyzed to be parallel to the $\{111\}$ slip plane, it can be speculated that the band structure is GNBs structure.
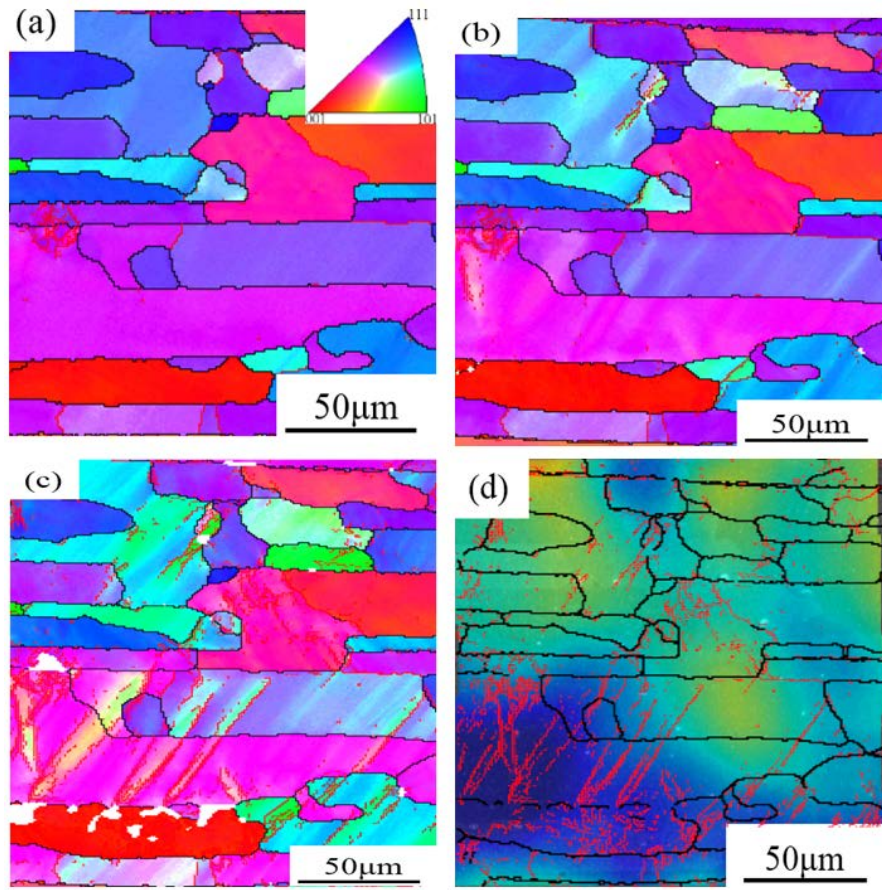

Fig.7 microstructure evolution under different bend radius: (a)unbend; (b) r=10mm; (c)

$\mathrm{r}=5 \mathrm{~mm}$; (d) strain combined with microstructure at $\mathrm{r}=5 \mathrm{~mm}$ (Black line mean large angle grain boundary, red line mean small angle grain boundary)

The results of the present study show that this band structure is related to the grain's orientation and strain [25]. The extended planar boundaries are termed GNB as they are believed to accommodate systematic slip differences in the grain. This is also in agreement with the typically alternating sign of the mis-orientation across adjacent GNBs[11].

\section{Conclusions}

The traditional MHPM and advanced DIC combined EBSD is used in study the strain and microstructure evolution during bending in compressive stress region. The conclusions of this study are as follows.

1. The traditional MHPM can only characterize the average strain change in micro-region, while DIC method can be used to characterize the strain micro-region on average and it can also represent the local strain tensor in the micro-region.

2. It is conclude that the amount of strain in grains are related to mis-orientation between the adjacent grains with EBSD and DIC methods of quasi-in situ experiments. The different mis-orientation has a distinctly different blocking effect on the slip of the dislocations. In general, the mis-orientation between adjacent grains is smaller with larger strain, while the mis-orientation between adjacent grains is larger with smaller strain.

3. The degree of grain division in the process of deformation is related to the strain in this region, a new microstructure such as band structure is observed in grains with larger strain. 


\section{Acknowledgements}

This work was supported by National Key Research and Development Program of China (No. 2016YFC0801901).

\section{References}

[1] B. Ahmed, S. Wu. Aluminum Lithium Alloys (Al-Li-Cu-X)-New Generation Material for Aerospace Applications. In: D. Yang, T.B. Zhang, Q. Luo (Eds), Applied Mechanics and Materials, 2014, pp. 104-111.

[2] R.J. Rioja, J. Liu, The Evolution of Al-Li Base Products for Aerospace and Space Applications, METALL MATER TRANS A, 43A(2012) 3325-3337.

[3] S.C. Wang, M.J. Starink, Precipitates and intermetallic phases in precipitation hardening Al-Cu-Mg-(Li) based alloys, INT MATER REV, 50(2005) 193-215.

[4] H. Kim, N. Nargundkar, T. Altan, Prediction of bend allowance and springback in air bending, J MANUF SCI E-T ASME, 129(2007) 342-351.

[5] R.J. Rioja, Fabrication methods to manufacture isotropic Al-Li alloys and products for space and aerospace applications, Materials Science and Engineering: A, 257(1998) 100-107.

[6] J. Sarkar, T.R.G. Kutty, D.S. Wilkinson, J.D. Embury, D.J. Lloyd, Tensile properties and bendability of T4 treated AA6111 aluminum alloys, Materials Science and Engineering: A, 369(2004) 258-266.

[7] T. W, Plastic surface strain mapping of bent sheets by image correlation, EXP MECH, 44(2004) 502-511.

[8] D.A. Hughes, N. Hansen, D.J. Bammann, Geometrically necessary boundaries, incidental dislocation boundaries and geometrically necessary dislocations, SCRIPTA MATER, 48(2003) 147-153.

[9] X. Huang, Grain Orientation Effect on Microstructure in Tensile Strained Copper, SCRIPTA MATER, 38(1998) 1697-1703.

[10] D.J. Jensen, Effects of orientation correlations on misorientation distributions in cold-deformed aluminium, Materials Science and Engineering: A, 234-236(1997) 762-765.

[11] G. Winther, D.J. Jensen, N. Hansen, Dense dislocation walls and microbands aligned with slip planes — theoretical considerations, ACTA MATER, 45(1997) 5059-5068.

[12] N.H. Qing Liu, Geometrically necessary boundaries and incidental dislocation boundaries formed during cold deformation, Scripta Metallurgica et Materialia, 32(1995) 1289-1295.

[13] J.A. WERT, Q. LIU, N. HANSEN, Dislocation boundaries and active slip systems, Acta Metallurgica et Materialia, 43(1995) 4153-4163.

[14] D.J. H, J.D. Juul, H. N, Large strain deformation structures in aluminium crystals with rolling texture orientations, Acta Metallurgica et Materialia, 42(1994) 3105-3114.

[15] X. Jin, B. Fu, C. Zhang, W. Liu, Strain localization and damage development in 2060 alloy during bending, International Journal of Minerals, Metallurgy, and Materials, 22(2015) 1313-1321.

[16] L. Mattei, D. Daniel, G. Guiglionda, H. Klöcker, J. Driver, Strain localization and damage mechanisms during bending of AA6016 sheet, Materials Science and Engineering: A, 559(2013) 812-821.

[17] R. Becker, An Analysis of Shear Localization During Bending of a Polycrystalline Sheet, Journal of Applied Mechanics, 59(1992) 491-496. 
[18] C.C. T, F.R. W, A.S. M, Applications of digital-image-correlation techniques to experimental mechanics, EXP MECH, 25(1985) 232-244.

[19] H. T. Huang. Study on Plastic Deformation Mechanism and Recrystallization Behavior of AZ31 Magnesium Alloy, Tsinghua University, 2013.

[20] W. M. Mao, Crystalline texture and anisotropy of metallic materials, Science Press, Beijing, 2002.

[21] T. Zhai, A.J. Wilkinson, J.W. Martin, A crystallographic mechanism for fatigue crack propagation through grain boundaries, ACTA MATER, 48(2000) 4917-4927.

[22] J. S. Pan, J. M. Tong, and M. B. Tian. Foundamental of Materials Science[B], Tinshua Press, Beijing, 1998.

[23] B. X Zhou, A Study of the preyielding microstructure in 3\%Si-Fe alloy, Acta Merallurgica Sin., (1983) 31-39.

[24] R. A, H.F. J, R.G. S, H. M. Recrystallization and related annealing phenomena, Elsevier, Netherlands, 2004.

[25] X. Jin, B. Fu, C. Zhang, W. Liu, Study of Dislocation Boundary Structure in Al-Li Alloy During Bending, Acta Metallurgica Sinica (English Letters), 28(2015) 1149-1155. 